DTP $95 / 5$

February, 1995

\title{
The Hamiltonian structure of the dispersionless Toda hierarchy
}

\author{
D.B.Fairlie \\ Dept. of Mathematical Sciences, University of Durham, \\ Durham, DH1 3LE, England 円. \\ I.A.B.Strachan \\ Dept. of Mathematics and Statistics, University of Newcastle, \\ Newcastle-upon-Tyne, NE1 7RU, England \&.
}

\begin{abstract}
The Hamiltonian structure of the two-dimensional dispersionless Toda hierarchy is studied, this being a particular example of a system of hydrodynamic type. The polynomial conservation laws for the system turn out, after a change of variable, to be associated with the axially symmetric solutions of the 3-dimensional Laplace equation and this enables a generating function for the Hamiltonian densities to be derived in closed form.
\end{abstract}

\section{Introduction}

In this paper the Hamiltonian structure of the integrable system

$$
\begin{aligned}
& S(x, t)_{t}=P(x, t)_{x}, \\
& P(x, t)_{t}=P(x, t) S(x, t)_{x},
\end{aligned}
$$

(or equivalently, the equation $(\log P)_{t t}=P_{x x}$ ) will be investigated. The reason for the interest in this system is two-fold. Firstly, it appears in various physical applications, for example:

- In the construction of self-dual Vacuum and Einstein-Weyl metrics [1].

- In topological field theory, as a solution to the WDVV equations [2].

- As the long-wave (or dispersionless) limit of the Toda equation [3, 4].

Secondly, despite its simplicity, it has the same generic properties as more complicated systems, so it can be used as a 'test bed' for various ideas. (See for example the recent paper of Mineev-Weinstein[5].) It is one of the simplest examples of an equation of hydrodynamic type that is, an equation of the form

$$
u_{t}^{i}=V_{j}^{i}(\mathbf{u}) u_{x}^{j}
$$

\footnotetext{
${ }^{1}$ e-mail: david.fairlie@durham.ac.uk

${ }^{2}$ e-mail: i.a.b.strachan@newcastle.ac.uk
} 
about which there is an extensive literature [6, 7]. Much of this, however, involves general theory, and there are comparatively few specific examples. The purpose of this paper is to derive concrete results for the dispersionless Toda system. Rather than study (11) directly, a transformation will be made to a new set $\{u, v\}$ of variables in which the equations are symmetric under the interchange of $u$ and $v$, this enabling results from the theory of homogeneous symmetric functions to be used. Explicitly, let $\{S, P\}$ be a solution to (1) and let $\{u, v\}$ be the roots of the quadratic

$$
z^{2}-S z+P=0
$$

In these new variables the original system (11) becomes

$$
\begin{aligned}
& u_{t}=u v_{x}, \\
& v_{t}=v u_{x},
\end{aligned}
$$

Note that if $u=v$ then this reduces to the dispersionless Korteweg-deVries equation.

This change of variables may be used to see the following property of any solution to (3). Given $\{u, v\}$ satisfying (3) a new solution is given by the roots of the quadratic

$$
z^{2}-(u+v+k) z+u v=0 .
$$

Moreover, one has a version of the 'theorem of permutability' -applying this transformation first with constant $k_{1}$ then with $k_{2}$ results in the same solution as applying the transformation with constant $k_{2}$ followed by that with $k_{1}$. The general solution to (1) or (3) may be found implicitly by performing a hodographic transformation. Specific examples have been calculated in [8] and the general form of the solution in [3].

Another motivation comes from the construction of integrable, relativistic system in $(1+1)$ dimensions. Any solution to (3) of the form

$$
\begin{aligned}
& u=\frac{f\left(\frac{\partial \phi}{\partial t} \frac{\partial \phi}{\partial x}\right)}{\left(\frac{\partial \phi}{\partial x}\right)^{2}} \\
& v=\frac{g\left(\frac{\partial \phi}{\partial t} \frac{\partial \phi}{\partial x}\right)}{\left(\frac{\partial \phi}{\partial x}\right)^{2}}
\end{aligned}
$$

(or, equivalently, a similarity solution) will result in such a system for $\phi$, with $x$ and $t$ being light-cone variables. The equations can be reduce to the single first order differential equation

$$
\frac{d Y}{d X}=\frac{Y(1+2 X-X Y)}{X(1+2 Y-X Y)}
$$

this being an Abel equation of the second kind. Despite the integrability of the original system (3) we have not been able to solve this equation, except in the case $Y=X$ (or $u=v$ ) which results in the well-known Born-Infeld equation for $\phi$ [9].

The rest of this paper is arranged as follows. In section 2 conservation laws will be derived for this system, together with a generating function for them, and in section 3 
the associated hierarchy will be studied. In section 4 these results will be used to study the Hamiltonian structure of this system and its hierarchy. These results follow from direct calculation and do not use the corresponding Lax pair. This Lax pair has a very simple solution, details of which may be found in the appendix. Various generalisations are presented in section 5 .

\section{Conservation Laws}

By direct calculation it is easy to find the first few polynomial conservation laws:

$$
\begin{aligned}
{[u+v]_{t} } & =[u v]_{x} \\
{\left[u^{2}+4 u v+v^{2}\right]_{t} } & =[2 u v(u+v)]_{x} \\
{\left[u^{3}+9 u v(u+v)+v^{3}\right]_{t} } & =\left[3 u v\left(u^{2}+3 u v+v^{2}\right)\right]_{x}
\end{aligned}
$$

and a general such law will be denoted by

$$
\mathcal{Q}_{n, t}=\mathcal{F}_{n, x}
$$

where $\mathcal{Q}_{n}$ (the charge density) is an $n^{\text {th }}$-order symmetric polynomial in $u$ and $v$, and $\mathcal{F}_{n}$ (the flux density) is an $(n+1)^{\text {th }}$-order symmetric polynomial in $u$ and $v$. The coefficients in $\mathcal{Q}_{n}$ are the squares of the binomial coefficients $B(n, r)$ :

$$
\mathcal{Q}_{n}=\sum_{r=0}^{n} B(n, r)^{2} u^{r} v^{n-r}
$$

and

$$
\mathcal{F}_{n}=\sum_{r=0}^{n} \frac{n-r}{r+1} B(n, r)^{2} u^{r+1} v^{n-r} .
$$

To prove this one assumes a general form for the symmetric polynomial $\mathcal{Q}$ and adjust the coefficients so that the $t$-derivative is a total $x$-derivative. These functions also satisfy the equations

$$
\begin{aligned}
\mathcal{F}_{n, u} & =v \mathcal{Q}_{n, v}, \\
\mathcal{F}_{n, v} & =u \mathcal{Q}_{n, u},
\end{aligned}
$$

from which follows the conservation law (4). The hierarchy of conservation laws may be combined into a single generating function

$$
\mathcal{Q}(\lambda)_{t}=\mathcal{F}(\lambda)_{x}
$$

so that the coefficient of $\lambda^{n}$ in its formal power series expansion is the conservation law (4). To show this it is first necessary to find a recursion relation amongst the above charge densities.

From the fact that $\mathcal{Q}_{n}$ and $\mathcal{F}_{n}$ are homogeneous polynomials in $u$ and $v$ of degree $n$ and $(n+1)$ respectively one obtains the following relations 


$$
\begin{aligned}
n \mathcal{Q}_{n} & =u \mathcal{Q}_{n, u}+v \mathcal{Q}_{n, v}, \\
(n+1) \mathcal{F}_{n} & =u \mathcal{F}_{n, u}+v \mathcal{F}_{n, v}
\end{aligned}
$$

and

$$
\begin{aligned}
& \mathcal{F}_{n+1, u}=(n+1)\left(\mathcal{F}_{n}+v \mathcal{Q}_{n}\right), \\
& \mathcal{F}_{n+1, v}=(n+1)\left(\mathcal{F}_{n}+u \mathcal{Q}_{n}\right) .
\end{aligned}
$$

Using these, together with (5), one easily obtains the following recursion relations:

$$
\begin{aligned}
\mathcal{Q}_{n+1} & =2 \mathcal{F}_{n}+(u+v) \mathcal{Q}_{n}, \\
(n+2) \mathcal{F}_{n+1} & =(n+1)\left[(u+v) \mathcal{F}_{n}+2 u v \mathcal{Q}_{n}\right]
\end{aligned}
$$

which results in the second order recurrence relation for the charge densities:

$$
(n+2) \mathcal{Q}_{n+2}-(2 n+3)(u+v) \mathcal{Q}_{n+1}+(n+1)(u-v)^{2} \mathcal{Q}_{n}=0 .
$$

This bears a strong resemblance to the recursion relation for Legendre polynomials. In fact, on defining

$$
\mathcal{Q}_{n}=(u-v)^{n} P_{n}\left(\frac{u+v}{u-v}\right)
$$

one obtains the Legendre recursion relation:

$$
(n+1) P_{n+1}(z)-(2 n+1) z P_{n}(z)+n P_{n-1}(z)=0
$$

and the first two conservations laws give $P_{1}(z)=1$ and $P_{2}(z)=\left(3 z^{2}-1\right) / 2$, the first two Legendre polynomials. Thus the charge densities may be expressed in terms of Legendre polynomials. Using the well-known generating function for these polynomials results in a generating function for the conservation laws:

$$
\frac{\partial}{\partial t}\left[\frac{1}{\sqrt{(u-v)^{2} \lambda^{2}-2(u+v) \lambda+1}}\right]=\frac{\partial}{\partial x}\left[\frac{\lambda(u+v)-1}{\sqrt{(u-v)^{2} \lambda^{2}-2(u+v) \lambda+1}}\right] .
$$

The form of this results suggest that one should define a new set of variables

$$
\begin{aligned}
r & =u-v, \\
\cos \theta & =\frac{u+v}{u-v} .
\end{aligned}
$$

In this systems the equation $\left(u \mathcal{Q}_{u}\right)_{u}=\left(v \mathcal{Q}_{v}\right)_{v}$ for the charge density becomes the axially symmetric Laplace equation in three dimensions. In terms of the inverse transformation

$$
\begin{aligned}
& u(x, t)=-r(x, t) \cos ^{2} \frac{\theta(x, t)}{2}, \\
& v(x, t)=r(x, t) \sin ^{2} \frac{\theta(x, t)}{2}
\end{aligned}
$$


the original system (3) becomes

$$
\begin{aligned}
& r_{t}=-\left(r^{2} / 2\right) z_{x}, \\
& z_{t}=\left(1-z^{2}\right) / 2 r_{x},
\end{aligned}
$$

where $z=\cos \theta$. The form of this suggest various generalizations, which will be investigated in section 5 .

It is clear that any solution to axially symmetric Laplace equation, besides the class of solutions solutions $r^{n} Q_{n}(\cos \theta)$ will yield conservation laws. In particular one also has three other basic solution, namely

$$
r^{-n-1} P_{n}(\cos \theta), \quad \quad r^{-n-1} Q_{n}(\cos \theta), \quad r^{n} Q_{n}(\cos \theta),
$$

where $Q_{n}(z)$ is the Legendre function of the second kind. If one wishes for conservation laws which remain regular as $r \rightarrow 0$ (i.e in the limit in which the dispersionless Toda system becomes the dispersionless $\mathrm{KdV}$ equation) then this excludes the first two. However the third remains and gives a family of non-polynomial conservation laws for (3), the first few being

$$
\begin{aligned}
{\left[\frac{1}{2} \log \left(\frac{u}{v}\right)\right]_{t} } & =\left[\frac{1}{2}(v-u)\right]_{x}, \\
{\left[\frac{1}{2} \mathcal{Q}_{1} \log \left(\frac{u}{v}\right)-(u-v)\right]_{t} } & =\left[\frac{1}{2} \mathcal{F}_{1} \log \left(\frac{u}{v}\right)+\frac{1}{4}(v-u)(v+u)\right]_{x}, \\
{\left[\frac{1}{2} \mathcal{Q}_{2} \log \left(\frac{u}{v}\right)-\frac{3}{2}\left(u^{2}-v^{2}\right)\right]_{t} } & =\left[\frac{1}{2} \mathcal{F}_{2} \log \left(\frac{u}{v}\right)+\frac{1}{6}(v-u)\left(v^{2}+10 u v+v^{2}\right)\right]_{x}
\end{aligned}
$$

(the logarithmic terms in the $(n+1)^{\text {th }}$ such conservation law are of the form $\frac{1}{2} \mathcal{Q}_{n} \log (u / v)$ and $\left.\frac{1}{2} \mathcal{F}_{n} \log (u / v)\right)$. Note that these are all antisymmetric under the interchange $u \leftrightarrow v$, and so vanish in the limit $r \rightarrow 0$.

\section{Generalised symmetries and commuting flows}

One fundamental property of an integrable system is the existence of an infinite family of commuting flows. For the system (3) it is straightforward to show that any such flow must be given by the system

$$
\begin{aligned}
& u_{t^{\prime}}=\mathcal{F} u_{x}+u \mathcal{Q} v_{x}, \\
& v_{t^{\prime}}=\mathcal{F} v_{x}+v \mathcal{Q} u_{x}
\end{aligned}
$$

where $\mathcal{F}$ and $\mathcal{Q}$ are any function of $u$ and $v$ (not necessarily symmetric) which satisfy the relations (5). Thus for each of the polynomial conservation laws derived in section 2 one has an associated flow labelled by the integer $n\left(=\right.$ degree of $\left.\mathcal{Q}_{n}\right)$,

$$
\begin{aligned}
& u_{t_{n}}=\mathcal{F}_{n} u_{x}+u \mathcal{Q}_{n} v_{x} \\
& v_{t_{n}}=\mathcal{F}_{n} v_{x}+v \mathcal{Q}_{n} u_{x}
\end{aligned}
$$


It is convenient to let $t_{0}=t, \mathcal{F}_{0}=0$ and $\mathcal{Q}_{0}=1$, so the original system corresponds to the $t_{0}$-flow. It follows from the general theory of generalised symmetries that the function $\mathcal{F} u_{x}+u \mathcal{Q} v_{x}$ and $\mathcal{F} v_{x}+v \mathcal{Q} u_{x}$ are characteristics for the original system, and that all these flows commute [10].

The charges are conserved with respect to the $t_{0}$-flow, and one might expect them also to be conserved with respect to the $t_{n}$-flow. This is indeed the case:

\section{Lemma}

$$
\frac{\partial \mathcal{Q}_{m}}{\partial t_{n}}=F_{n} \frac{\partial Q_{m}}{\partial x}+Q_{n} \frac{\partial F_{m}}{\partial x} .
$$

Furthermore, the right hand side is a derivative;

$$
\frac{\partial \mathcal{Q}_{m}}{\partial t_{n}}=\frac{\partial \Delta(m, n)}{\partial x}
$$

where

$$
\Delta(m, n)=\frac{\mathcal{F}_{m, u} \mathcal{F}_{n+1, v}+\mathcal{F}_{m, v} \mathcal{F}_{n+1, u}}{(n+1)(m+n+1)} .
$$

\section{Proof}

The first form of the equation (10) follows straightforwardly from (9). The second is most easily seen by the introduction of a potential.

From (7) it is easy to change variables to obtain the Cauchy-Riemann like equations

$$
\begin{aligned}
\frac{\partial \mathcal{F}_{n}}{\partial \mathcal{F}_{m}} & =\frac{\partial \mathcal{Q}_{n}}{\partial \mathcal{Q}_{m}} \\
\frac{\partial \mathcal{F}_{n}}{\partial \mathcal{Q}_{m}} & =P \frac{\partial \mathcal{Q}_{n}}{\partial \mathcal{F}_{m}}
\end{aligned}
$$

Introducing a potential

$$
\mathcal{F}_{n}=\frac{\partial \Delta(m, n)}{\partial \mathcal{Q}_{m}}, \quad \mathcal{Q}_{n}=\frac{\partial \Delta(m, n)}{\partial \mathcal{F}_{m}}
$$

the equation (10) simply becomes

$$
\frac{\partial \mathcal{Q}_{m}}{\partial t_{n}}=\frac{\partial \Delta(m, n)}{\partial Q_{m}} \frac{\partial Q_{m}}{\partial x}+\frac{\partial \Delta(m, n)}{\partial F_{m}} \frac{\partial F_{m}}{\partial x}=\frac{\partial \Delta(m, n)}{\partial x}
$$

A more detailed analysis shows that the potential $\Delta(m, n)$ is given by (11). This makes use of equations (5, (6) and (77). In the case $m=1, \mathcal{F}_{1}=S, \mathcal{Q}_{1}=P$ the potential is simply given by $\Delta(1, n)=\frac{\mathcal{F}_{n+1}}{n+1}$ 


\section{The Hamiltonian structure of the hierarchy}

A system (2) of hydrodynamic type is said to be Hamiltonian if there exists a Hamiltonian $H=\int d x h(\mathbf{u})$ and a Hamiltonian operator

$$
\hat{A}^{i j}=g^{i j}(\mathbf{u}) \frac{d}{d x}+b_{k}^{i j}(\mathbf{u}) u_{x}^{k}
$$

which defines a skew-symmetric Poisson bracket on functionals

$$
\{I, J\}=\int d x \frac{\delta I}{\delta u^{i}(x)} \hat{A}^{i j} \frac{\delta J}{\delta u^{j}(x)}
$$

which satisfies the Jacobi identity and which generates the system

$$
u_{t}^{i}=\left\{u^{i}(x), H\right\}
$$

Dubrovin and Novikov [6] proved necessary and sufficient conditions for $\hat{A}^{i j}$ to be a Hamiltonian operator in the case when $g^{i j}$ is not degenerate. These are:

a) $\mathbf{g}=\left(g^{i j}\right)^{-1}$ defines a Riemannian metric,

b) $b_{k}^{i j}=-g^{i s} \Gamma_{s k}^{j}$, where $\Gamma_{s k}^{j}$ is the Christoffel symbol generated by $\mathbf{g}$,

c) the Riemann curvature tensor of $\mathbf{g}$ vanishes.

The system (2) may then be written as

$$
u_{t}^{i}=\left(g^{i s} \nabla_{s} \nabla_{j} h\right) u_{x}^{j}
$$

where $\nabla$ is the covariant derivative generated by $\mathbf{g}$. Thus to find the Hamiltonian structure for the hierarchy (9) one needs to find the metric and the Hamiltonians.

One approach is to first diagonalise the system, there being the simple formulae for the metric coefficients for diagonal systems [7]:

$$
\frac{\partial_{i} V_{j}}{\left(V_{i}-V_{j}\right)}=\frac{1}{2} \partial_{i} \log g_{j j}
$$

where $V_{j}^{i}(\mathbf{u})=V_{j}(\mathbf{u}) \delta_{j}^{i}$ (no sum). This diagonalisation is achieved by the transformation:

$$
\begin{aligned}
U & =\sqrt{u}+\sqrt{v} \\
V & =\sqrt{u}-\sqrt{v} .
\end{aligned}
$$

While this enables one to find the metric, the formulae are actually more elegant in the $\{u, v\}$ coordinates.

\section{Theorem}

The Hamiltonian structure is given by the zero-curvature metric

$$
\mathbf{g}=2 \frac{d u}{u} \frac{d v}{v}
$$

and the Hamiltonians are given by 


$$
H_{n}=\int d x h_{n}(\mathbf{u})
$$

where $h_{n}=(n+1)^{-2} \mathcal{Q}_{n+1}$.

\section{Proof}

The metric is clearly non-degenerate and flat, and the only non-zero Christoffel symbols are $\Gamma_{11}^{1}=-u^{-1}$ and $\Gamma_{22}^{2}=-v^{-1}$ (here $u^{1}=u$ and $u^{2}=v$ ). Hence, on expanding (13) one obtains the following equations for $h_{n}$

$$
\begin{aligned}
\mathcal{F}_{n} & =u v \frac{\partial^{2} h_{n}}{\partial u \partial v} \\
\mathcal{Q}_{n} & =\frac{\partial}{\partial v}\left[v \frac{\partial h_{n}}{\partial v}\right] \\
& =\frac{\partial}{\partial u}\left[u \frac{\partial h_{n}}{\partial u}\right]
\end{aligned}
$$

With $h_{n}=(n+1)^{-2} \mathcal{Q}_{n+1}$ these reduce to identities derived in section 2 and 3 . Hence the result.

This show the elegance of the $\{u, v\}$ coordinate system: in terms of $\{P, S\}$ or $\{r, \theta\}$ the metric is considerably more complicated.

\section{Proposition}

The Hamiltonians $H_{n}$ are time independent and in involution with respect to the Poisson bracket given by $\mathbf{g}$.

\section{Proof}

The result follows from the formulae already derived.

$$
\begin{aligned}
\frac{d H_{n}}{d t_{m}} & =(n+1)^{-2} \int d x \frac{\partial \mathcal{Q}_{n+1}}{\partial t_{m}} \\
& =(n+1)^{-2} \int d x \frac{\partial \Delta(n+1, m)}{\partial x} \\
& =0
\end{aligned}
$$

under suitable boundary conditions. The second results follows from the use of the same formula:

$$
\begin{aligned}
\left\{H_{m}, H_{n}\right\} & =\int d x \frac{\partial h_{m}}{\partial u^{i}} \hat{A}^{i j} \frac{\partial h_{n}}{\partial u^{j}} \\
& =(n+1)^{-2} \int d x \mathcal{F}_{n} \frac{\partial \mathcal{Q}_{m+1}}{\partial x}+\mathcal{F}_{n} \frac{\partial \mathcal{F}_{m+1}}{\partial x} \\
& =(n+1)^{-2} \int d x \frac{\partial \Delta(m+1, n)}{\partial x} \\
& =0
\end{aligned}
$$


The factor $(n+1)^{-2}$ may, without loss of generality, be absorbed into a redefinition of the times $t_{n}$. One therefore obtains the following generating function for the Hamiltonians:

$$
\mathcal{H}(\lambda)=\int d x h(\lambda)
$$

where

$$
h(\lambda)=\frac{1}{\lambda \sqrt{(u-v)^{2} \lambda^{2}-2(u+v) \lambda+1}}-\frac{1}{\lambda} .
$$

\section{Generalizations}

The appearance of Legrendre polynomials suggests that there might be similar systems with conservation laws given in terms of more general polynomials. The system

$$
\begin{aligned}
& S_{t}=P_{x}, \\
& P_{t}=P^{\omega} S_{x}
\end{aligned}
$$

may be transformed into the system

$$
\begin{aligned}
& u_{t}=(u v)^{\alpha} u v_{x} \\
& v_{t}=(u v)^{\alpha} v u_{x}
\end{aligned}
$$

where $\alpha=(\omega-1) /(2-\omega)$ (the case $\omega=2$ having to be treated separately). By an analogous proceedure to that outlined above, one can show that this has conservation laws expressible in terms of Gegenbauer polynomials $T_{n}^{\alpha}$, so when $\alpha=0$ these collapse to Legendre polynomials. The Hamiltonian structure is also similar; this is given by the metric

$$
\mathbf{g}=2 \frac{d u}{u^{\alpha+1}} \frac{d v}{v^{\alpha+1}}
$$

and the Hamiltonians are directly related to the charges, as before.

More generally still, one can look for polynomial conservation laws for the system

$$
\begin{aligned}
& r_{t}=r^{a} q(z) z_{x} \\
& z_{t}=r^{b} p(z) r_{x}
\end{aligned}
$$

this being a direct generalisation of equation (8). Looking for charge densities and fluxes of the form $\mathcal{F}=r^{c} w(z), \mathcal{Q}=r^{d} y(z)$ results in the constraints $a-b=2$ and $c=b+d-1$, and the self-adjoint differential equation

$$
\frac{d}{d z}\left(p(z) \frac{d y}{d z}\right)-d(d+a-1) y=0
$$


for $y(z)$. Assuming that this has regular points at 0,1 and $\infty$ results in a hypergeometric equation and constraints on the otherwise free functions $p(z)$ and $q(z)$. To get polynomials solutions requires $d$ to be a non-negative integer, and so finally the charge densities are

$$
\mathcal{Q}_{n}=r_{2}^{n} F_{1}(-n, n+a-1, c ; z) \text {, }
$$

where ${ }_{2} F_{1}$ is the hypergeometric series. In terms of the $u, v$ variables one obtains:

$$
\begin{aligned}
& u_{t}=u^{a-c} v^{c-1} v_{x} \\
& v_{t}=u^{a-c-1} v^{c} u_{x}
\end{aligned}
$$

There is, however, considerable degeneracy in this system, with apparently different systems being connected via a change in variable. Other systems may be obtained, for example, by assuming that (14) is a confluent hypergeometric function, and this contains a family of charges given by Laguerre polynomials.

\section{Acknowledgements}

We would like to thank Chris Athorne for suggesting the introduction of a potential in the proof of the lemma in section 3. I.A.B.S. would like to thank the University of Newcastle for a Wilfred Hall Fellowship.

\section{Appendix}

The results in this paper have been obtained by direct calculation; no use has been made of any Lax equation. In this appendix the Lax equation will be studied, and the solution to the associated linear problem derived. The system (11) is a special case of the more general equation

$$
\partial_{t} \partial_{t^{\prime}} \log P=\partial_{x}^{2} P
$$

known as the Boyer-Finley or $S U(\infty)$ Toda equation. This is itself a reduction of the equations for an anti-self-dual Ricci-flat metric. Physically the system (1) describes antiself-dual Ricci-flat metrics with two commuting Killing vectors, and so any Lax pair will fall into the class studies by Tod and Ward [1]. One possible Lax pair for the BoyerFinley equation (there are other, equivalent, formulations [12]) was given by Ward [1], and imposing the symmetry $\partial_{t}=\partial_{t^{\prime}}$ gives a Lax pair for (1).

Explicitly, a Lax pair for the dispersionless Toda system (11) is given by

$$
\begin{aligned}
& \mathcal{L}_{0}=\partial_{t}-L_{f}-\xi L_{e^{+}}, \\
& \mathcal{L}_{1}=\xi\left(\partial_{t}+L_{f}\right)+L_{e^{-}},
\end{aligned}
$$

where $\left[L_{f}, L_{g}\right]=L_{\{f, g\}}$ with $\{f, g\}=f_{y} g_{x}-f_{x} g_{y}$ and $e^{ \pm}=P^{\frac{1}{2}}(x, t) \exp ( \pm y), f=\frac{1}{2} S(x, t)$. Then the integrability condition $\left[\mathcal{L}_{0}, \mathcal{L}_{1}\right]=0$ for this otherwise overdetermined linear system 


$$
\begin{aligned}
& \mathcal{L}_{0} \Psi=0, \\
& \mathcal{L}_{1} \Psi=0,
\end{aligned}
$$

gives, on equating the coefficients of the various powers of $\xi$, the spectral parameter, to zero, the system $P_{t}=P S_{x}, S_{t}=P_{x}$.

The solution $\Psi$ to the linear system may be found in the form of a power series

$$
\Psi=\sum_{n=0}^{\infty} \psi_{n}(x, t) \exp (n y) \xi^{n} .
$$

The $\psi_{n}$ are not unique - one can multiple by an arbitrary holomorphic function of $\xi$ and this will change all the coefficients, but not the integrability conditions, this corresponding to a gauge freedom. This yields an infinite number of recursion relations

$$
\begin{aligned}
\partial_{x} \theta_{n+1} & =P^{n} \partial_{t}\left(P^{-n} \theta_{n}\right), \\
\partial_{t} \theta_{n+1} & =P^{n+1} \partial_{x}\left(P^{-n} \theta_{n}\right),
\end{aligned}
$$

where

$$
\theta_{n}(x, t)=\psi_{n} P^{\frac{n}{2}}
$$

together with the initial conditions $\partial_{x} \theta_{0}=\partial_{t} \theta_{1}=0$. The first few solutions to these equations are

$$
\begin{aligned}
& \theta_{0}=k_{0}, \\
& \theta_{1}=k_{1} \\
& \theta_{2}=-k_{1} S+k_{2}, \\
& \theta_{3}=k_{1}\left(S^{2}-P\right)-2 k_{2} S+k_{3} .
\end{aligned}
$$

Here the $k_{i}$ are constants. Using the above-mentioned gauge freedom one can set $k_{0}=0$ and $k_{i}=0$ for $i \geq 2$, so that, for $n \geq 1, \theta_{n}$ is an $(n-1)^{\text {th }}$ polynominal in $u$ and $v$. After a few more iterations one finds the relation

$$
\theta_{n+1}+S \theta_{n}+P \theta_{n-1}=0, \quad n \geq 2
$$

appears to work, and this can be proved by induction (again using the the gauge freedom to set various constants to zero). Solving this relation gives

$$
\theta_{n}=(-1)^{n+1} k_{1}\left(\frac{u^{n}-v^{n}}{u-v}\right)
$$

and hence $\psi_{n}$. Summing the power series is straightforward:

$$
\Psi=\frac{k_{1}}{\sqrt{u v}} \frac{1}{\xi^{-1} \exp (-y)+\left[\sqrt{\frac{u}{v}}+\sqrt{\frac{v}{u}}\right]+\xi \exp (+y)}
$$

where $\tilde{\xi}=\xi \exp y$. In all this $y$ plays an auxiliary rôle. This may be removed in favour of $\tilde{\xi}=\xi \exp (+y)$, but this will introduce terms involving $\partial_{\tilde{\xi}}$ in the Lax pair. 


\section{References}

[1] R.S.Ward, Class. Quantum Grav. 7 (1990) L95, J. Geom. Phys. 8 (1992) 317.

[2] I.M.Krichever, Commun. Pure Applied Math. 47 (1994) 437.

[3] M.Saveliev, On the integrability problem of the continuous long wave approximation of the Toda lattice, preprint ENSL, Lyon, 1992.

[4] J. Hoppe and Q-Han Park, Infinite Charge Algebra of Gravitational Instantons, Phys. Lett. B321 (1994) 333-337.

[5] M. B. Mineev-Weinstein, Conservation Laws in Field Dynamics or Why Boundary Motion is Exactly Integrable? Los Alamos preprint Ms-B258 (1995)

[6] B.A.Dubrovin and S.P.Novikov, Soviet Math. Doklady 27 (1983) 665.

[7] S.P.Tsarev, Soviet Math. Doklady 31 (1985) 488, Math. USSR Izves. 37 (1991) 397.

[8] Y.Kodama, Phys. Lett. A147 (1990) 477.

[9] D.B.Fairlie and J.A.Mulvey, J. Phys. A27 (1994) 1317.

[10] P.J.Olver, Applications of Lie Groups to Differential Equations, (Springer, New York, 1986).

[11] K.P.Tod and R.S.Ward, Proc. R. Soc. A386 (1979) 411.

[12] K.Takasaki and T.Takebe, Lett. Math. Phys. 23 (1991) 205. 